\title{
HIP ARTHOPLASTY IN A FORESTER AFTER NUMEROUS TICK BITES
}

\author{
ZBIGNIEW SZYMCZAK ${ }^{1}$, TADEUSZ PŁUSA ${ }^{2}$, PAWEŁ BARANOWSKI ${ }^{1}$, and JAROSŁAW KRAWCZYK ${ }^{1}$ \\ ${ }^{1}$ Mazovian Center of Rehabilitation STOCER, Konstancin-Jeziorna, Poland \\ Department of Neurorthopedics \\ ${ }^{2}$ Medical Faculty of the Lazarski University in Warsaw, Warsaw, Poland
}

\begin{abstract}
Arthritis is one of the most important symptoms of late-stage borreliosis, observed in approx. $60 \%$ of untreated patients with Lyme disease. In several percent of them, this manifestation is of interest to orthopedic surgeons. The authors present a case of a patient, working as a forester, who underwent left hip replacement arthroplasty having contracted a Borrelia burgdorferi infection in the past, and who was hospitalized 12 years before in the infectious ward due to suspected neuroborreliosis for which he was treated with Ceftriaxone $2 \times 2.0 \mathrm{~g}$ intravenously for 4 weeks. At that time, a tomographic examination of the spinal cord revealed herniated nuclei at levels L4/L5 and L5/S1, which filled the left lateral recess, narrowing the intervertebral holes and compressing the nerve roots on the left side of L4 and L5. In September 2019, total hip replacement in the patient's left hip joint was performed. It is concluded that an active role of neuroborreliosis in this process of hip joint destruction may be suggested. Int J Occup Med Environ Health. 2021;34(5):693-9
\end{abstract}

Key words:

Lyme disease, rehabilitation, occupational disease, gouty arthritis, hip replacement arthroplasty, acquired joint deformity

\section{INTRUDUCION}

Borreliosis (Lyme disease), on the one hand, is the most common tick-borne illness in the Northern Hemisphere, with multisystem symptoms and dermatologic, cardiac [1], and neurologic [2] complications [3]. On the other hand, arthritis is one of the most important symptoms of latestage borreliosis, observed in approx. $60 \%$ of untreated patients with Lyme disease [4]. In several percent of them, this manifestation is of interest to orthopaedic surgeons [5].

Due to Borrelia burgdorferi spirochetes implementation, following an infected tick bite, an inflammatory response is induced in the synovial tissue with synovial hypertrophy, vascular proliferation, and infiltration of inflammatory cells [6]. The released proinflammatory cytokines, immune complexes, complement and recruited neutrophils are present in the synovial fluid. Moreover, Borrelia burgdorferi is able to induce chondrocytes to produce matrix metalloproteinases which are responsible for degradation of extracellular matrix proteins, proteoglycans, and collagen [7]. The elevated concentrations and activities of biologically active mediators, including the above-mentioned, may be responsible for cartilage damage and joint destruction observed in the late stage of borreliosis [5].

For this reason, patients after prior arthritis due to Lyme disease should be under constant specialist control, because in the late stage of the disease it may be necessary to undergo surgery in the form of total joint replacement.

Received: August 7, 2020. Accepted: February 1, 2021.

Corresponding author: Zbigniew Szymczak, Mazovian Centre of Rehabilitation STOCER, Department of Neuroorthopedics, Wierzejewskiego 12, 05-510 Konstancin-Jeziorna, Poland (e-mail: zibi_sz@wp.pl). 
The authors present a case of a patient who underwent left hip replacement arthroplasty having contracted a Borrelia burgdorferi infection in the past.

\section{CASE REPORT}

The patient, a 42-year-old man, was admitted to surgery for left hip arthroplasty. The reason for qualification to surgical treatment was pain he had experienced in the left hip joint, increasing for 2 years with a progressive limitation of movement. The patient also reported a past infection with Borrelia and gout.

The patient was working as a forester [8] and, following numerous tick bites, 12 years ago he was hospitalized in the infectious ward due to suspected neuroborreliosis, which was manifested by dizziness, general weakness and weakness of sensation, as well as pain in the left iliac region. During the hospitalization, the patient underwent serological tests for a potential Borrelia infection, yielding the following results:

- in the blood: IgG ELISA (Biomedica, Piaseczno, Poland) - positive: $45.7 \mathrm{BBU} / \mathrm{ml}$ (a positive result from 11.0 BBU/ml) and IgM ELISA (Biomedica) - negative; - in the cerebrospinal fluid: IgG ELISA (Biomedica) negative and IgM ELISA (Biomedica) - negative.

In the performed lumbar puncture, it was found that the cerebrospinal fluid was colorless and clear, and the following parameters were determined: cytosis -3 , protein $0.33 \mathrm{~g} / \mathrm{l}$, glucose $-3.00 \mathrm{mmol} / \mathrm{l}$, lactic acid - $1.09 \mathrm{mmol} / \mathrm{l}$, chlorides - $119.3 \mathrm{mmol} / \mathrm{l}$, Nonne-Apelta plus/minus test, and Pandy's test - one plus.

In addition, determinations made by real-time polymerase chain reaction (PB-Bob-03) for the presence of a Borrelia burgdorferi sensu lato infection also gave a negative result. Subsequent examinations (performed in 2012) revealed only the presence of the $\mathrm{p} 41$ antigen in the $\mathrm{IgG}$ fraction in the Western blot test, and in 2014 a borderline result was obtained (IgG 24.06 RU/ml).

It should be added that, during the patient's stay in the infectious ward, a tomographic examination of the spinal cord revealed herniated nuclei at levels L4/L5 and L5/S1, which filled the left lateral recess, narrowing the intervertebral holes and compressing the nerve roots on the left side of L4 and L5. Features of high compression on the dural sack at both levels, and a significant reduction of the canal reserve, were also shown. In addition, the presence of degenerative changes in the intervertebral discs with a decrease in their height, as well as shallow lordosis of the lumbar spine, were described.

In the period from the patient's hospital treatment to his admission to the orthopedic department, he remained under occassional specialist control.

Despite inconclusive results, the patient was given Ceftriaxone (Biotriakson, Polpharma, Starogard Gdański, Poland) $2 \times 2.0$ g intravenously for 4 weeks, and bifidobacterium + lactobacillus (Trilac, Krotex Pharm, Warsaw, Poland) and Diclofenacum natricum (Majamil, Polpharma, Starogard Gdański, Poland) orally after antibiotic treatment. Subsequently, he was referred to a Lyme disease outpatient clinic for further observation.

On the basis of his medical documentation from the hospitalization period and a history of numerous tick bites while he was working as a forester (a ranger), the patient obtained a decision under article $104 \S 1$ and 2 of the Code of Administrative Procedure, and article 5 of Capt. 4a of the Act of 14 March 1985, from the State Sanitary Inspection regarding the diagnosis of an occupational disease, and more specifically Lyme disease. In Poland, Lyme disease is included in the list of occupational diseases affecting foresters. In addition, $20 \%$ of health damage caused by the effects of that occupational disease was determined (a decision of the Department of Social Security certifying doctor).

For the first time, the patient reported to the orthopedic outpatient clinic in 2017 due to experiencing pain in his left hip joint, gradually increasing for a year. The physical examination revealed a limited range of motion in the left hip joint. There were some degenerative changes of this hip joint - III/IV ${ }^{\circ}$ on the Kellgren-Lawrence 


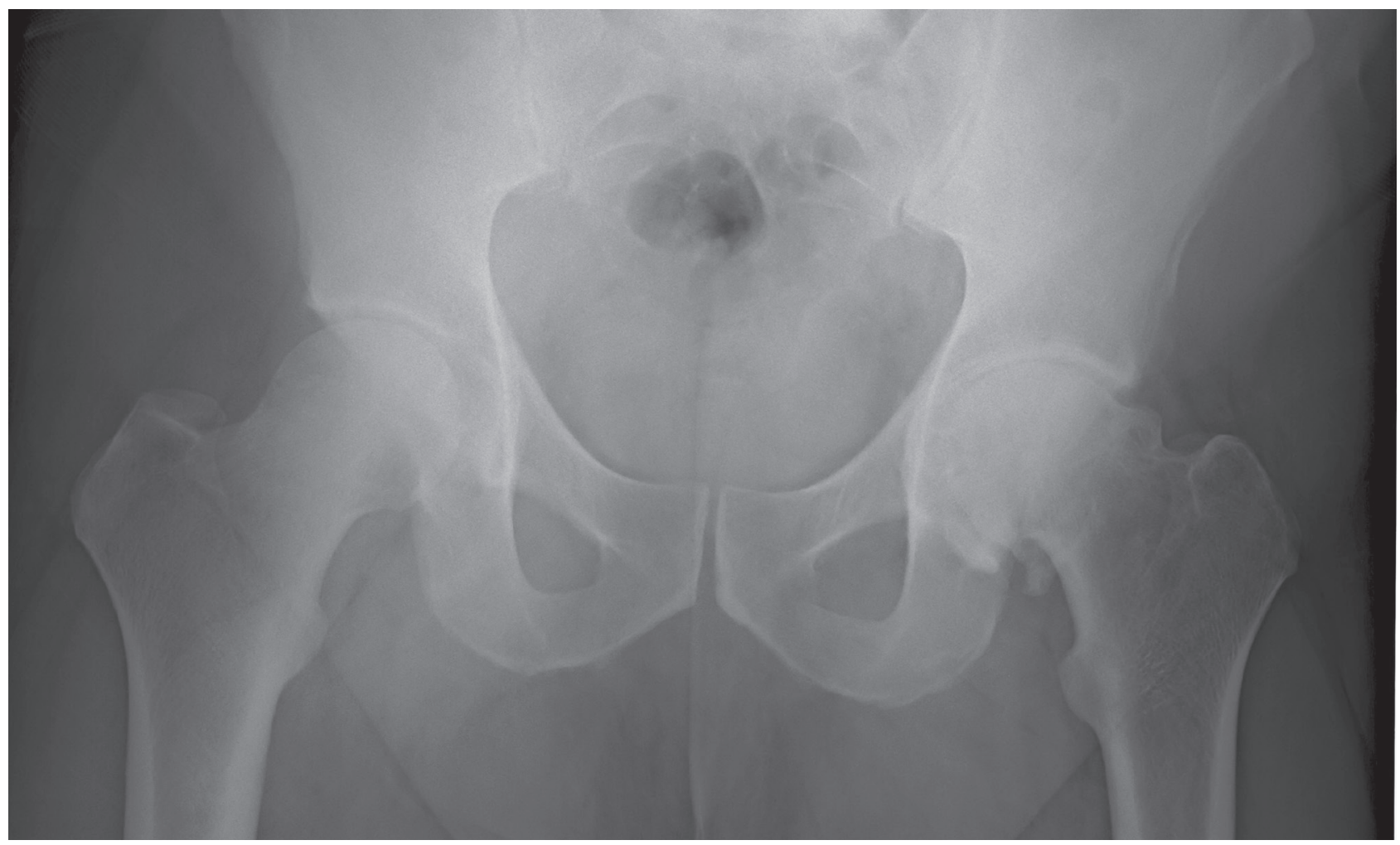

Figure 1. Degenerative changes in the left hip joint - III/IV ${ }^{\circ}$ on the Kellgren-Lawrence Scale, in the radiological image, in 2017

Scale [9], in the radiological image (Figures 1 and 2). No degenerative changes were revealed in the right hip joint. Based on the above data, the patient was qualified for left hip arthroplasty. However, given his young age and professional reasons, the patient did not decide to promptly undergo the surgery. Instead, he received intra-articular injections of hyaluronic acid $(16 \mathrm{mg} / 2 \mathrm{ml}$, Synvisc, Sanofi Aventis, Warsaw, Poland) at semi-annual intervals. After each injection, pain relief of the left hip joint was obtained. During subsequent outpatient visits, treatment of hyperuricemia (the highest serum uric acid levels of $8.2 \mathrm{mg} \%$ ) was also adjusted.

In September 2019, total hip replacement in the patient's left hip joint was performed, using Metha-type endoprosthesis (B. Braun Aesculap Chifa, Nowy Tomyśl, Poland). A screw-in socket and monoblock - a titanium stem with hydroxyapatite coating - were used to ensure good bio- compatibility of the implants and to facilitate the process of osseointegration between the implant and the bone. The inlay of the acetabulum and the head of the endoprosthesis were made of BIOLOX®delta zirconia ceramics. This type of an endoprosthesis is especially recommended for young people due to the relatively small amount of resected bone tissue, which results from the use of a short stem. On the other hand, the ceramic surfaces enabling the endoprosthesis to move are made of the hardest materials available today, which ensures a small volume of abrasion products, and also minimizes the inflammatory reaction, which is a cause of the "around a foreign body" type reaction.

The histopathological examination (No. H9030) of the hip joint capsule obtained during the surgery showed the presence of foci with single neutrophils in the internal synovium layer from the joint cavity, in the absence of 
a) b) c)

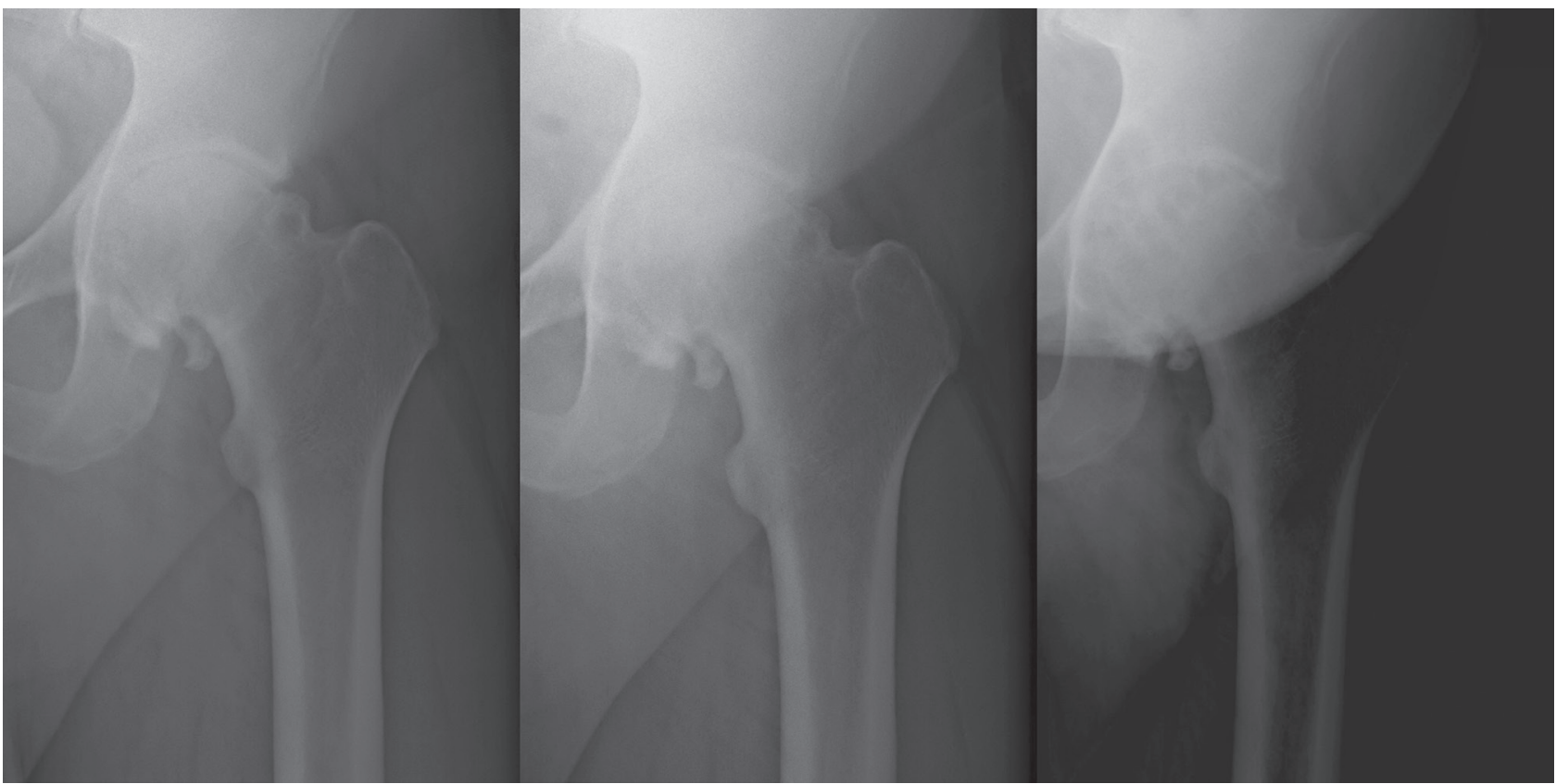

Figure 2. Gradual destruction of the left hip joint in: a) 2016, b) 2017, and c) 2018

lymphocytic and plasmacytar infiltrates. Exudative lesions and other inflammatory infiltrations were not disclosed in the outer part of the joint capsule despite the fact that X-ray features of the degeneration of the left hip joint were described (Figure 3a). The revealed changes were difficult to differentiate from secondary changes resulting from osteoarthritis, especially since no characteristics of the Lyme disease-related inflammation of the perivascular mixed inflammatory infiltrates or changes in the walls of blood vessels were revealed.

The postoperative course was uncomplicated. During the patient's stay in the ward, in 2 subsequent examinations a tendency to decrease the $\mathrm{C}$-reactive protein (CRP) concentration was observed. The wound was healed by primary intention.

In November 2019, the patient reported to the hospital-based outpatient clinic due to a postoperative reaction. There were some small point reactions in the wound line corresponding to the location of the subcutaneous sutures. Cultures were collected and empirical antibiotic treatment was initiated (Cloxacillinum [Syntarpen, Polfa Tarchomin, Warsaw, Poland] orally, in accordance with hospital antibiotic policy). In the radiological examination, no signs of loosening or periprosthetic infection were revealed (Figure 3b). Due to the linear calcifications above the acetabulum, additional tests were performed to determine the concentrations of calcium, phosphorus, alkaline phosphatase, vitamin $\mathrm{D}_{3}$ and CRP in the serum, which were normal except for a significant decrease in the serum vitamin $\mathrm{D}_{3}$ concentration $(7 \mathrm{ng} / \mathrm{ml})$. Oral supplementation of vitamin $\mathrm{D}_{3}$ was, therefore, recommended. Methicillin-sensitive Staphyloccus aureus was cultured in the wound material and antibiotic therapy was continued. The healing process was completed within a week. During the subsequent outpatient visit at the end of December 2019, an efficient gait, without limping, was found. The radiological examination showed ectopic ossification in the acetabular region (Figure 3c). For this reason, the patient was urgently referred to a rehabilitation ward. Control sera of patients with a Borrelia burgdorferi infection were obtained in September 2019, yielding nega- 
a)

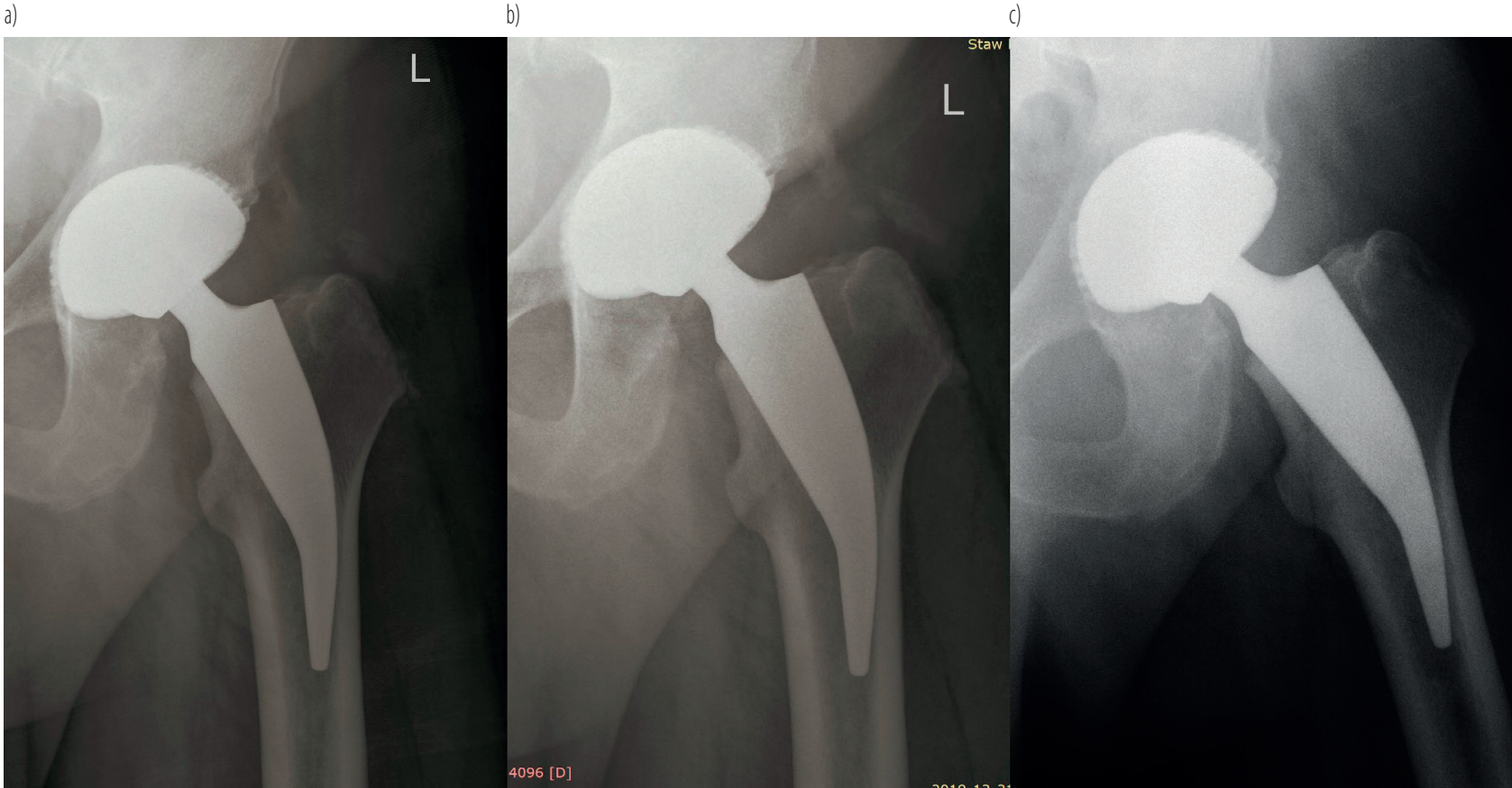

Figure 3. Radiological examination of the left hip joint: a) after the surgery, b) during the first control visit, and c) during the second control visit

tive $\operatorname{IgM}(0.2)$ and $\operatorname{IgG}(0.3)$ antibody titers, but the presence of OspC antigen was revealed in Western blot test (p41, test No. 8221/2019/EK-3/1 performed at the National Institute of Hygiene in Warsaw).

The last outpatient visit (June 2020) revealed the restoration of almost a full range of motion in the left hip. There was no progression of ectopic ossification in the X-ray examination. The patient returned to his previous work as a forester.

The patient's consent for the publication of this medical data was given orally. All published medical data is anonymous.

\section{DISCUSSION}

In the discussed case, the clinical and radiological picture, from the orthopedic point of view, does not present much controversy. Doubts can be raised only by the young age of the patient who underwent hip arthroplasty, but the severity of the degenerative disease did not allow any other treatment. Nevertheless, the etiopathogenesis of such advanced changes at young age involving only 1 hip joint seems to raise some doubts.
Three main causes of joint destruction can be considered. The first is arthritis in the course of Lyme disease. The arguments for such a reason is the coincidence of the spirochete infection experienced. The clinical involvement of Lyme disease, described in the natural course of the disease, is more similar to autoimmune diseases than to septic arthritis. Lyme arthritis usualy involves a single large joint, most commonly the knee, with involvement in up to $90 \%$ of affected patients [10]. After a month-long course of antibiotic treatment, no spirochete was found in the joint in a genetic test. At the same time, joint changes could persist, resembling reactive arthritis. The location of the changes speaks against such etiopathogenesis - in Lyme disease changes most often (in about 70\%) concern the knee joint and they are exudated in this joint. On the other hand, an exudate in the hip joint is difficult to find in a physical examination. When long and thin needles are used for the injection of hyaluronic acid into the hip joint, only in the case of a large amount of the exudate and under high pressure, a backflow of synovial fluid is found to occur. This was not 
the case with this patient. At the same time, the patient reported multi-site "roaming" joint pain, typically found in Lyme disease or post-Lyme disease.

Uric acid may be considered the second possible joint damage factor. Although the patient did not report episodes of typical gouty arthritis (gout attack) associated with the precipitation of uric acid crystals in the joint fluid, prolonged serum uric acid levels $>6.8 \mathrm{mg} \%$ might cause uric acid salt deposition in soft tissues, leading to their gradual damage.

The third reason to consider is the possibility of arthropathy resulting from neurological disorders. The factors damaging peripheral nerves include lumbar discopathy causing stenosis of the intervertebral holes, which causes damage to the spinal nerves. This etiological factor should be taken into account due to the CT vertebral stenosis, larger on the left side, which corresponds to the side of the hip joint affected by degenerative changes. At the same time, during Lyme disease, peripheral nerve damage is often found in the mechanism of both direct axonal damage and the stimulation of autoimmune processes that cause nerve damage. The lack of clinically apparent neurological disorders, in the form of sensory disorders or paresis, speaks against this etiopathogenesis. However, a relatively small pain sensation in the presence of extensive radiological changes within the joint resemble this picture of neurogenic artopathy, e.g., in Charcot's disease.

It also seems possible that the active process of the Borrelia burgdorferi infection exhibited by the patient treated in accordance with accepted recommendations for neuroborreliosis may have caused changes in the already morphologically changed hip joint. According to recommendations, Lyme arthritis should be successfully managed with oral antibiotics but in adults with neurologic involvement a 2-4-weeklong course of intravenously cefuroxime axetil $(500 \mathrm{mg}$ twice daily) is acceptable but discussible [11]. In the presented case, the patient was treated with ceftriaxon (Biotriakson, Polpharma) $2 \times 2.0$ intravenously for 4 weeks, which is more efficient and selective to spirochetes [12]. This treat- ment was successful and the neurologic symptoms disappeared, but the inflammation process activated by Borrelia burgdorferi in the hip joint was still going on. The confirmation of the immunological changes may be the fact that the detected OspC $\mathrm{p} 41$ antigen is still present.

The treatment of degenerative changes of the hip in this patient was typical and involved hip replacement with an endoprosthesis. The etiology of these changes, which is not fully understood, requires special attention during further treatment, because of the possibility of some unusual complications, e.g., problems with wound healing which can be associated with the attenuation of the immune system by spirochetes. The relationship between low vitamin $\mathrm{D}_{3}$ concentration and atypical ossification around the acetabulum is not fully explained either.

It has been documented in neuroborreliosis patients that an extracellular actin-scavenger system is composed of gelsolin and vitamin D binding protein, and can cause cell damage and toxicity of surrounding cells [13]. Moreover, isolated corticotropic insufficiency has been described in $21 \%$ of patients with previous neuroborreliosis [14]. The observed endocrine dysfunctions and vitamin D deficiency after Lyme neuroborreliosis could be a possible explanation for musculoskeletal complaints [15].

These observations are very interesting but it remains unclear if the decresed concentration of vitamin $\mathrm{D}_{3}$ in the serum of the analyzed patient provides evidence for possible spirochete survival despite the negative $\operatorname{IgG}$ and $\operatorname{IgM}$ results, or indicates some other active process caused by the immune reaction of an immunologically changed tissue. Anyway, the obtained orthopedic results were accepted by the patient but they require long-term observation.

\section{CONCLUSIONS}

Based on the above scenarios, it is possible to conclude that there are many factors damaging the hip joint, each of which would not lead to the development of the disease, but with their synergic action, there is an effect of mutual 
strengthening. This exceeds the body's compensatory abilities and leads to some degenerative changes in the hip joint. An active role of neuroborreliosis in this process of hip joint destruction may be suggested.

\section{REFERENCES}

1. Scheffold N, Herkommer B, Kandolf R, May AE. Lyme carditis - diagnosis, treatment and prognosis. Dtsch Arztebl Int. 2015;112(12):202-8, https://doi.org/10.3238/arztebl.2015.0202.

2. Rauer S, Kastenbauer S, Fingerle V, Hunfeld KP, Huppertz HI, Dersch R. Lyme Neuroborreliosis. Dtsch Arztebl Int. 2018;115(45):751-6, https://doi.org/10.3238/arztebl.2018.0751.

3. Obel N, Dessau RB, Krogfelt KA, Bodilsen J, Andersen NS, Møller JK, et al. Long term survival, health, social functioning, and education in patients with European Lyme neuroborreliosis: nationwide population based cohort study. BMJ. 2018;361:k1998, https://doi.org/10.1136/bmj.k1998.

4. Biesiada G, Czepiel J, Leśniak MR, Garlicki A, Mach T. Lyme disease: review. Arch Med Sci. 2012;8(6):978-82, https://doi. org/10.5114/aoms.2012.30948.

5. Smith BG, Cruz AI Jr, Milewski MD, Shapiro ED. Lyme disease and the orthopaedic implications of lyme arthritis. J Am Acad Orthop Surg. 2011;19(2):91-100, https://doi. org/10.5435/00124635-201102000-00004.

6. Brouwer MAE, van de Schoor FR, Vrijmoeth HD, Netea MG, Joosten LAB. A joint effort: The interplay between the innate and the adaptive immune system in Lyme arthritis. Immunol Rev. 2020;294(1):63-79, https://doi.org/10.1111/imr.12837.

7. Bramwell KK, Mock K, Ma Y, Weis JH, Teuscher C, Weis JJ. $\beta$-Glucuronidase, a Regulator of Lyme Arthritis Severity, Modulates Lysosomal Trafficking and MMP-9 Secretion in Response to Inflammatory Stimuli. J Immunol. 2015;195(4):164756, https://doi.org/10.4049/jimmunol.1500212.
8. Richard S, Oppliger A. Zoonotic occupational diseases in forestry workers - Lyme borreliosis, tularemia and leptospirosis in Europe. Ann Agric Environ Med. 2015;22(1):43-50, https://doi.org/10.5604/12321966.1141368.

9. Kellgren JH, Lawrence JS. Radiological assessment of osteo-arthrosis. Ann Rheum Dis. 1957;16(4):494-502, https:// doi.org/10.1136/ard.16.4.494.

10. Bamm VV, Ko JT, Mainprize IL, Sanderson VP, Wills MKB. Lyme Disease Frontiers: Reconciling Borrelia Biology and Clinical Conundrums. Pathogens. 2019;8(4):299, https://doi. org/10.3390/pathogens8040299.

11. Cameron DJ, Johnson LB, Maloney EL. Evidence assessments and guideline recommendations in Lyme disease: the clinical management of known tick bites, erythema migrans rashes and persistent disease. Expert Rev Anti Infect Ther. 2014;12(9):1103-35, https://doi.org/10.1586/14787210. 2014.940900.

12. Stażyk K, Czepiel J, Gumulska M, Garlicki A, Biesiada G. Analysis of cases of Lyme arthritis in patients hospitalized in Infectious Diseases Department, University Hospital in Cracow. Folia Med Cracov. 2019;59(1):5-14.

13. Pachner AR, Steiner I. Lyme neuroborreliosis: infection, immunity, and inflammation. Lancet Neurol. 2007;6(6):54452, https://doi.org/10.1016/S1474-4422(07)70128-X.

14. Bennett JM, Reeves G, Billman GE, Sturmberg JP. Inflammation-Nature's Way to Efficiently Respond to All Types of Challenges: Implications for Understanding and Managing "the Epidemic" of Chronic Diseases. Front Med (Lausanne). 2018;5:316, https://doi.org/10.3389/fmed.2018.00316.

15. Abbasi M, Hashemipour S, Hajmanuchehri F, Kazemifar AM. Is vitamin D deficiency associated with non specific musculoskeletal pain? Glob J Health Sci. 2012;5(1):107-11, https://doi.org/10.5539/ghhs.v5n1p107.

This work is available in Open Access model and licensed under a Creative Commons Attribution-NonCommercial 3.0 Poland License - http://creativecommons.org/ licenses/by-nc/3.0/pl/deed.en. 